\title{
Corrigendum: Topological crystalline insulators in the SnTe material class
}

Timothy H. Hsieh, Hsin Lin, Junwei Liu, Wenhui Duan, Arun Bansil \& Liang Fu

Nature Communications 3:982 doi:10.1038/ncomms1969 (2012); Published 31 Jul 2012; Updated 21 May 2013

In the Discussion section of this Article, we incorrectly claimed that an in-plane magnetic field will generate Dirac mass terms for the surface states. Instead, the in-plane magnetic field merely shifts the location of the Dirac points. The $k \cdot p$ Hamiltonian in the presence of an in-plane field $\left(B_{1}, B_{2}\right)$ (in the local basis defined in the Article) is

$$
\begin{gathered}
H_{\mathrm{s} f, \mathrm{~B}}=v_{\perp} k_{1} s_{2}-v_{\|} k_{2} s_{1}+g_{1} \mu_{\mathrm{B}} B_{1} s_{1}+g_{2} \mu_{\mathrm{B}} B_{2} s_{2} \\
=v_{\perp}\left(k_{1}+\frac{g_{2} \mu_{\mathrm{B}} B_{2}}{v_{\perp}}\right) s_{2}-v_{\|}\left(k_{2}-g_{1} \mu_{\mathrm{B}} \frac{B_{1}}{v_{\|}}\right) s_{1}
\end{gathered}
$$

where $\mu_{\mathrm{B}}$ is the Bohr magneton and $g_{1,2}$ is the $g$-factor of Dirac surface states. 